\title{
Influence of Partner Selection on Functional Differentiation: Emergence of Diversity by Isolated Interaction and Preference Change
}

\author{
Saori Iwanaga \\ Department of Maritime Safety Technology, Japan Coast Guard Academy, 5-1 Wakaba \\ Kure, Hiroshima 7378512, Japan \\ Akira Namatame \\ Computer Science Department., National Defense Academy, 1-10-20 Hashirimizu \\ Yokosuka, Kanagawa 2398686, Japan \\ E-mail: s-iwanaga@jcga.ac.jp
}

\begin{abstract}
When examining collective behavior, we focus on the interactions of individuals. We must also consider the microscopic level, where individual agent decisions occur, and the macroscopic level, where collective behavior can be observed. Collective behavior is both interesting and difficult because aggregate outcomes must be evaluated within environmental constraints. The performance of a collective system strongly depends on the type of interaction and heterogeneity of agent preference.

In this study, relative to the emergence of diversity, we found that two different learning processes are required. In addition, we found that isolated interactions are required for the emergence of diversity. However, the utility of less clustered interactions is greater than that of isolated interactions. We found that isolated interactions are effective for the emergence of diversity. Once diversity emerges sufficiently, less clustered interactions are effective relative to the utility of each agent and the entire population.
\end{abstract}

Keywords: collective behavior, diversity, payoff matrix, isolated interaction, preference.

\section{Introduction}

Studies into multi-robot and multi-agent simulations began in the late 1980s. Reynolds ${ }^{1}$ shows that collective behavior occurs when three rules are used simultaneously by Boids and that the collective behavior is a complex system. Sims ${ }^{2}$ study of virtual block creatures and Terzopoulos ${ }^{3}$ study of artificial fish provide example of emerged physical or functional characteristics. Kubo et al. ${ }^{4}$ demonstrate the emergence of unique individuals in a competition for food between ant colonies. However, individual heterogeneity is often ignored in most studies ${ }^{5}$. For example, a recent study investigated the formation by multi robots. In a multirobot system, if heterogeneous robots exist, such robots are removed $^{6}$. There are limits to the adaptation performance of multi robot systems with heterogeneous robots, and computer simulations rarely reproduce emerged diversity in the population.

When examining collective behavior, we focus on the interactions of individuals. We must also describe two different levels, i.e., the microscopic level, where decisions of individual agents occur, and the macroscopic level where collective behavior can be observed $^{7}$. Schweitzer ${ }^{8}$ shows that interaction at the microscopic level results in complex behavior at the macroscopic level. Collective behavior is interesting and difficult because the aggregate outcome must be evaluated within environmental constraints. The performance of a collective system strongly depends on the type of interaction the heterogeneity in agent ${ }^{9}$ preference. 
Axelrod et al. ${ }^{5,10}$ show that as individual agents experience more diverse contacts, the system can become less diverse. As interaction patterns become less clustered, giving individuals the experience of interacting with more "distant" others, information moves more rapidly throughout. The world becomes so "small" that the sociological "islands" vanish, unable to keep their local ways and remain untouched by events elsewhere. Early innovations spread too fast, and variety that can provide later improvements is lost. This is called premature convergence. The ideal breeding ground for novel live-forms seems to be an archipelago or a network of mountain valleys.

Sandholm el.al ${ }^{10}$ presents a dynamic analysis of the evolution of preferences in a strategic environment. Dynamics run at two speeds at once: while natural selection slowly reshapes the distribution of preferences, players quickly learn to behave as their preferences dictate. They establish the existence and uniqueness of the paired trajectories of society's preferences and aggregate behavior. While aggregate behavior adjusts smoothly in equilibration games, in coordination games aggregate behavior can jump discretely in an instant of evolutionary time.

In this study, we clarify how diversity emerges in a population.

\section{Model}

There is an increasing amount of research into bounded rationality, and the hypotheses employed in such research reflect the ability of each agent to receive partial information from other agents through interaction $^{12}$. Our model can be interpreted in a similar manner; however, we intend to combine the hypotheses of adaptation and local interactions to model evolutionary processes. The first hypothesis reflects the limited ability of each agent to receive, make a decision, and act on information obtained though interactions. The second interpretation is that agents choose the best action. We formalize these ideas in a model with a finite agent population in which agents are repeatedly matched within a given game period, which we describe. There are many parameters to consider such as payoff structure, localization, the shadow of the future, and the number of agents. Here we examine payoff structure and localization.

We adopt the payoff matrix shown in Table 1, which describes the outcome of interactions between agents. In this matrix, if both agents chose the same strategy, they can obtain a positive payoff $1-\theta_{i}$ or $\theta_{i}$; otherwise they receive nothing, where $0 \leq \theta_{i} \leq 1$. This is a coordination game.

There are many situations where interacting agents can benefit from coordinating actions. Coordination usually implies that increased effort by some agents encourages the remaining agents to follow suit, which gives rise to multiplier effects. For examples, where coordination is important for trade alliances and the choice of compatible technologies or conventions, such as software or program language. These situations can be modeled as coordination games; in which agents are expected to select the majority strategiy ${ }^{13,14}$. However, traditional game theory, does not consider how agents know which equilibrium should be realized if a coordination game has multiple and equally plausible Pareto-ranked equilibria ${ }^{15,16}$. This limitation is all the more surprising in games with common goal because one expects that players will coordinate to the Paretodominant equilibrium ${ }^{17}$. Game theory has been also unsuccessful in explaining how agents should behave to improve an equilibrium situation ${ }^{18}$.

Considering that payoff parameters $\theta_{i}$ of both agents are 0 , it is Nash equilibrium and Pareto optimum that both of two agents choose same S1. Nash equilibrium is a state in which the most suitable strategy is selected by all agents. The Pareto optimum is a state in which there is no room to increase the benefit of an agent without reducing benefits of other agents.

Table 1. Payoff matrix of agent Ai.

\begin{tabular}{cccc}
\hline & & \multicolumn{2}{c}{ Behavior of other agent } \\
\cline { 2 - 4 } & & $\mathrm{S} 1$ & $\mathrm{~S} 2$ \\
\hline Behavior of & $\mathrm{S} 1$ & $1-\theta_{i}$ & 0 \\
agent $\mathrm{Ai}$ & $\mathrm{S} 2$ & 0 & $\theta_{i}$ \\
\hline
\end{tabular}

\subsection{Agent preference}

Payoff parameter $\theta_{i}$ characterizes the agent preference. We deal with the expression of heterogeneity of agents. In the population, each agent has different payoff parameters $\theta_{i}$ and $1-\theta_{i}$ as shown in Table 1 . If payoff parameter $1-\theta_{i}$ is greater than $\theta_{i}$ of agent $\mathrm{Ai}$, the agent prefers $\mathrm{S} 1$. If parameter $1-\theta_{i}$ of agent $\mathrm{Ai}$ is less than parameter $\theta_{i}$, the agent prefers $\mathrm{S} 2$. For example, if the payoff parameter $\theta_{i}$ of agent $\mathrm{Ai}$ is less than 0.5 , the agent prefers S1, otherwise, agent Ai prefers S2. 


\subsection{Social network}

In many situations, agents are not assumed to be knowledgeable as to correctly guess or anticipate other agents' strategies, or they are less sophisticated in that they do not know how to calculate the best resonse ${ }^{12}$. The hypotheses we employ in the local interaction model reflect limited ability to receive, decide, and act on information obtained through interactions. Each agent receives partial information from other agents through interaction ${ }^{13}$. In addition, each agent chooses an optimal strategy based on information from other agents. The consequences of agent behavior affects agents with whom they are not directly linked, and agents gradually learn the strategy of the population.

A lattice model is used to clarify the ecological characteristic of living beings. Nowak ${ }^{19}$ and Axelrod ${ }^{10}$ deal with situation in which each agent faces a social dilemma, and they indicate the importance of the structure of the space to emerge corporation in society. We consider the lattice structure shown in Fig. 1, in which each agent interacts with only neighbors of the agent. In this paper, we deal with the von Neumann neighborhood model. In the von Neumann neighborhood model, each agent interacts with its nearest four agents.

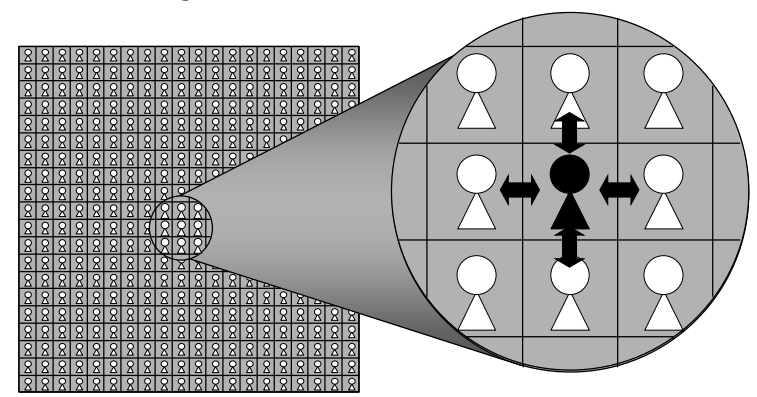

Fig. 1. von Neumann neighborhood model

\subsection{Behavior decision}

Agents do not know the behavior their partners will take, but they do know the previous behavior if partners. For agent $\mathrm{Ai}$, the proportion of agents having selected $\mathrm{S} 1$ at time $\mathrm{t}$ is defined by $p_{i}(t)\left(0 \leq p_{i}(t) \leq 1\right)$ in neighbors of agent Ai. Each agent can take the best response against the behavior the partners took by calculating the expected payoff. Agent Ai compares the average expected utilities of $\mathrm{S} 1$ with $\mathrm{S} 2$ at time $\mathrm{t}$, which are given as flows:

$$
\begin{aligned}
& \bar{U}_{i}(S 1, t)=p_{i}(t)\left(1-\theta_{i}\right), \\
& \bar{U}_{i}(S 2, t)=\left(1-p_{i}(t)\right) \theta_{i} .
\end{aligned}
$$

Then, the agent maximize their utility. Agent $\mathrm{Ai}$ chooses $\mathrm{S} 1$ if $\bar{U}_{i}(S 1, t)>\bar{U}_{i}(S 2, t)$ or chooses $\mathrm{S} 2$ if $\bar{U}_{i}(S 1, t)<\bar{U}_{i}(S 2, t)$ at the next time step. The decision rule of agent $\mathrm{Ai}$ at time $t+1$ is described as the following two cases depending on idiosyncratic parameter $\theta_{i}$.

If $p_{i}(t)>\theta_{i}$, agent Ai chooses $\mathrm{S} 1$;

otherwise $p_{i}(t)<\theta_{i}$, agent Ai chooses S2. (2)

This is the best response rule. When $p_{i}(t)=\theta_{i}$, we assume that the agent chooses the same previous behavior. We define the right-hand side of (2) as the threshold of agent Ai. The crucial point for dealing with diversity in the population is this threshold. Agent behavior differs according to the threshold. Moreover, the proportion of agents that chose S1 among the partners $p_{i}(t)$ of the agent differs. Thus, agent decision can differ. In other word, the payoff matrix and the world affect agent behavior and form collective behavior in the population. We define collective behavior $p(t)$ as the proportion of agents that have selected $\mathrm{S} 1$ in the entire population at time $t$.

\subsection{Moves for new neighbors}

If an agent wants to obtain a high payoff, it is effective to move to another site and change partners. In studies 14,20 , the agent changes partners based on partner properties. It is often difficult to observe the properties of a partner, however, it is easy to observe behaviors. We cannot observe an internal attribute from outside. We can only observer an action that appears as an internal attribute. In addition, we often treat the action of an agent given a predetermined internal attribute. However, it is reasonable that the action taking by an agent forms an internal attribute. In this study, each agent changes partners based on behaviors.

Each agent has parameter $\mu$. If the average payoff of agent Ai obtained by interaction with four partners is greater than or equal to $\mu$, agent $\mathrm{Ai}$ remains the site. Otherwise agent $\mathrm{Ai}$ moves to another site. The rule for changing partners is as follows, where $k=1,2$.

If $\bar{U}_{i}(S k, t) \geq \mu$, agent Ai remains at the site; otherwise $\bar{U}_{i}(S k, t)<\mu$, agent $\mathrm{Ai}$ moves to another site.

Some agents want to move to another site in the population. The pairs of agents are matched randomly and change sites each other. In addition, all agents that want to change can change partners simultaneously. Initially, an agent in a new site adopts the behavior of 
the agent previously in the site because it is not familiar with the site. Then, the agent decides the behavior according to Eq. (2).

If agent can move, agents can contact diverse agents and interaction is less clustered. On the other hand, when agents cannot move to another site, interaction is clustered and corresponds to isolated interaction.

\subsection{Self-reinforcement of preference}

In this study, we clarify how diversity emerges in a population. Each agent can update the payoff parameter through interaction. We define this as "selfreinforcement of preference." If agent Ai has selected $\mathrm{S} 1 \mathrm{~T}$ times in succession, agent $\mathrm{Ai}$ increases payoff $1-\theta_{i}$ for S1 by delta $\Delta$ and decreases payoff $\theta_{i}$ for S2 by delta $\Delta$. Otherwise, if agent Ai selected S2 T times in succession, agent $\mathrm{Ai}$ increases payoff $\theta_{i}$ for $\mathrm{S} 2$ by delta $\Delta$ and decreases payoff $1-\theta_{i}$ for $\mathrm{S} 1$ by delta $\Delta$.

Table 2. Updated payoff matrix of agent Ai after selecting S1

\begin{tabular}{cccc}
\multicolumn{3}{c}{ T time steps in succession } \\
\cline { 3 - 4 } & & \multicolumn{2}{c}{ Behavior of other agent } \\
\cline { 2 - 4 } Behavior of & $\mathrm{S} 1$ & $1-\theta_{i}+\Delta$ & $\mathrm{S} 2$ \\
agent Ai & $\mathrm{S} 2$ & 0 & $\theta_{i}-\Delta$ \\
\hline
\end{tabular}

The reinforced payoff at the next time is shown in Tables 2 and 3.

Table 3. Updated payoff matrix of agent Ai after selecting S2 $\mathrm{T}$ time steps in succession

\begin{tabular}{cccc}
\hline & & \multicolumn{2}{c}{ Behavior of other agent } \\
\cline { 2 - 4 } & & $\mathrm{S} 1$ & $\mathrm{~S} 2$ \\
\hline Behavior of & $\mathrm{S} 1$ & $1-\theta_{i}-\Delta$ & 0 \\
agent $\mathrm{Ai}$ & $\mathrm{S} 2$ & 0 & $\theta_{i}+\Delta$ \\
\hline
\end{tabular}

\section{Simulation}

\subsection{Simulation settings}

We arranged agents for a $50 \times 50$ area (2500 agents) with no gap. Note that four corners or ends of an area connect to an opposite side. Initially, we set payoff parameter $\theta_{i}$ to 0.5 for all agents and we set $\mu$ to 0.5 based on a preliminary experiment.

At initial time step, we set the initial collective behavior $p(0)$, which is the proportion of agents having chosen $\mathrm{S} 1$ in the whole population that select $\mathrm{S} 1$, from 0.0 to 1.0 at intervals of 0.125 . Here, we assume that an agent makes a random selection. Each agent makes its decision depending on the best response rule for each time step, and then the collective behavior $p(t)$ changes. We simulated 100 trials per initial collective behavior and investigated final collective behavior $p^{*}$ (at time step 1000).

We simulated three (Table 4). For the M-0.01 case, delta $\Delta$ is 0.01 and the agent can change partners. In this case, agent interactions are less clustered. For the S-0.01 case, delta $\Delta$ is also 0.01 ; however, the agent cannot change partners and remains at the site. In this case, all agents stay at the site and always interact with four stable neighbors, i.e., isolated interaction. For the M-0.1 case, delta $\Delta$ is 0.1 and the agent can change partners. Self-reinforcement with delta $\Delta$ of 0.1 results in faster learning than delta $\Delta$ of 0.01 .

Table 4. Simulation patterns

\begin{tabular}{lrcr}
\hline & & \multicolumn{2}{c}{ Change partners } \\
\cline { 2 - 4 } & & Movable & Stay \\
\hline \multirow{2}{*}{ delta $\Delta$} & 0.01 & M-0.01 & S-0.01 \\
\cline { 2 - 4 } & 0.1 & M-0.1 & --- \\
\hline
\end{tabular}

\subsection{Criteria}

We evaluated collective behavior using four criteria, i.e., stability, arrangement, efficiency and equity. Stability is the path-dependency of collective behavior. We also evaluated final collective behavior $p^{*}$, which represents the proportion of agents that select $\mathrm{S}$, starting from some initial collective behavior $p(0)$. For arrangement of collective behavior, we investigated the behavior of all agents finally. We compared the behavior to the arrangement of agent preferences.

Efficiency was evaluated by average utility, i.e., the measure of the desirability of the macro level. Here, high efficiency, i.e., high average utility, means that agents in the population obtain high utilities as a whole. We define the proportion of agents $g(u)$ who obtained utility $u$ per partner at the last interaction. Efficiency $U$ is given as follows;

$$
U=\int_{0}^{1} u g(u) d u
$$

If two populations have the same efficiency, the utility distributions differ. One population may demonstrated and another population may demonstrate inequity. Equity measures desirability at the micro level. In economics, inequity is often calculated by the Lorenz curve. The Lorenz curve represents the cumulative proportion of total utility, which is cumulated to the 
proportion at level $x$ starting with the poorest agents. Using $\mathrm{g}(u)$, the Lorenz curve $L(x)$ is given by $L(x)=$ $\int_{0}^{x} \tau g(\tau) d \tau / \int_{0}^{1} \tau g(\tau) d \tau$. Equity $E$ is obtained by the area under the Lorenz curve as follows;

$$
E=2 \int_{0}^{1} L(x) d x
$$

where parameter $x$ satisfies $x=\int_{0}^{w} g(\tau) d \tau$ and $w$ is the obtained utility.

\subsection{Simulation results}

The simulation results are shown in Figs. 2, 3 and 4. For case $\mathrm{M}-0.01$, the arrangement of the agents' final behaviors are shown in Fig. 2(1), and the arrangement of agent final positions are shown in Fig. 2(2). When the initial collective behavior $p(0)$ is 0.125 , all agents select S1 at last (Fig. 2(1)(a)) and the final collective behavior $p^{*}$ becomes 0.0 . When the initial collective behavior $p(0)$ is 0.125 , the payoff parameter $\theta_{i}$ of all agents changes 1.0 at last (Fig. 2(2)(a)). When the initial collective behavior $p(0)$ is 0.875 , all agents select $\mathrm{S} 2$ at last (Fig. 2(1)(c)), and the final collective behavior $p^{*}$ becomes 1.0. When the initial collective behavior $p(0)$ is 0.875 , the payoff parameter $\theta_{i}$ of all agents changes 0.0 at last (Fig. 2(2)(c)). Diversity does not emerged in the population.

On the other hand, When the initial collective behavior $p(0)$ is 0.5 , half of the agents select $\mathrm{S} 1$ and other half select S2 at last (Fig. 2(1)(b)), and the final collective behavior $p^{*}$ becomes 0.5 . When the initial collective behavior $p(0)$ is 0.5 , the payoff parameter $\theta_{i}$ of half of the agents changes to 0.0 and payoff parameter of the other half changes 1.0 at last (Fig. 2(2)(b)). We found that diversity emerged in the population. Moreover, comparing with Figs. 2(1)(b) and 2(2)(b), agents with payoff parameter 0.0 select $\mathrm{S} 1$ according to preferences. Agents with payoff parameter 1.0 select S2, according to preferences. Segregation occurs and agents with the same preference can interact. We define segregated interaction as selective interaction.

In cases S-0.01 (Fig. 3) and M-0.1(Fig. 4), the properties of assignment of the agents at last are similar to those of case M-0.01. However, in case S- 0.01 , when the initial collective behavior $p(0)$ is 0.125 or 0.875 , minority behavior survives (Figs. 3(1)(a) and 3(1)(c)). Because the agent cannot change partners, local behavior may persist. With regard to preferences, a slight but noticeable diversity emerges in the population
(Figs. 3(2)(a) and 3(2)(c)). In case S-0.01, When the initial collective behavior $p(0)$ is 0.5 , it is difficult to form selective interaction (Fig. 3(1)(b)), unlike case M0.01 .

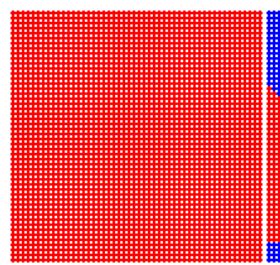

(a) $p(0)=0.125$

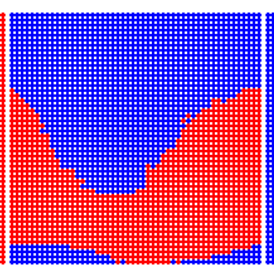

(b) $p(0)=0.5$

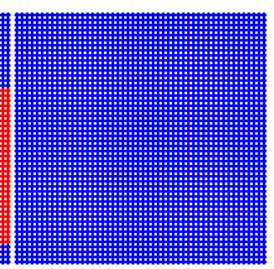

$p(0)=0.875$
(1) Behaviors of agents (examples)

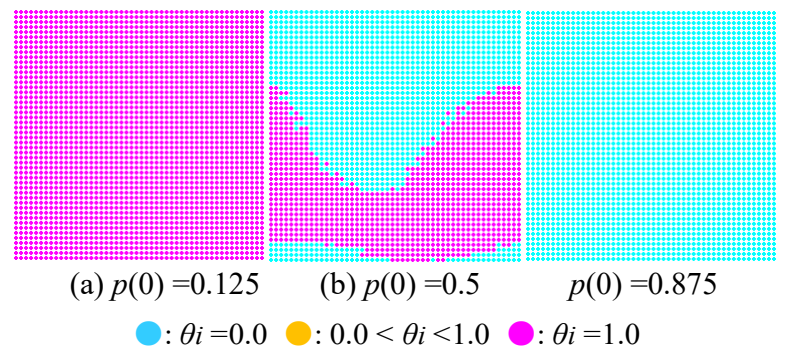

(2) Preferences of agents (examples)

Fig. 2. Assignment of agents (case M-0.01)

In cases M-0.01 and M-0.1, when the initial collective behavior $p(0)$ is 0.5 , selective interaction occurs (Figs. 2(1)(b) and 4(1)(b))).We found that, by changing partners, selective interaction of agents with the same preference occurs. When delta $\Delta$ is 0.01 , segregation is clear, and selective interaction is formed when delta $\Delta$ is 0.1 .

The stability of collective behavior is shown in Fig. 5. In this figure, the $x$-axis represents the initial collective behavior $p(0)$ and the $y$-axis represents the average final collective behavior $p^{*}$.

For case $\mathrm{M}-0.01$, we found that the final collective behavior depends on the initial collective behavior $p(0)$. If the initial collective behavior is greater than or equal to 0.75 , collective behavior converges to 1.0 and all agents select $\mathrm{S} 1$ in all trials. Otherwise, if the initial collective behavior is less than or equal to 0.25 , collective behavior converges to 0.0 and all agents select S2 in all trials. In other word, the majority behavior survives and the minority behavior disappears. However, if the initial collective behavior is between 0.375 and 0.625 , the collective behavior becomes intermediate, i.e., 0.0 or 1.0 , depending on the trial and the initial collective behavior. If the initial collective 
behavior $p(0)$ is 0.375 , the final collective behavior $p^{*}$ becomes 0.05 . If the initial collective behavior $p(0)$ is 0.5 , the final collective behavior $p^{*}$ becomes 0.54 . If the initial collective behavior $p(0)$ is 0.625 , the final collective behavior $p^{*}$ becomes 0.97 . Then, behaviors $\mathrm{S} 1$ and $\mathrm{S} 2$ can coexist.

For case M-0.1, the collective behavior also depends on the initial collective behavior $p(0)$, however, the property of the final collective behavior $p^{*}$ is more moderate than M- 0.01 , i.e., the graph curve for M- 0.1 is gentler than that of M-0.01.

For case S-0.01, the collective behavior depends on the initial collective behavior $p(0)$, and the final collective behavior $p^{*}$ is the most moderate and the graph curve for S- 0.01 is the gentlest among the three cases.

In Fig. 5, for reference, we show the simulation results of the default cases, wherein all agents do not self-reinforce. The default $M$ is the case where the agent can change partners. With default $M$, the agent can change its partners, and there is a distinct initial value dependency $(0.0$ or 1.0$)$ depending on the initial collective behavior $p(0)$. Default $\mathrm{S}$ is the case whereby the agent cannot change partners. Default $\mathrm{S}$ is similar to S-0.01, however, agents self-reinforce in this case. In addition, behaviors S1 and S2 can coexist.

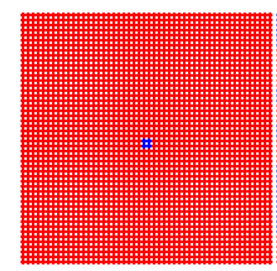

(a) $p(0)=0.125$

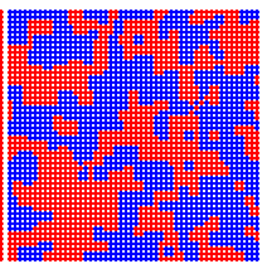

(b) $p(0)=0.5$

: $\mathrm{S} 1$

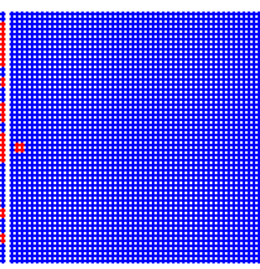

$p(0)=0.875$
(1) Behaviors of agents (examples)

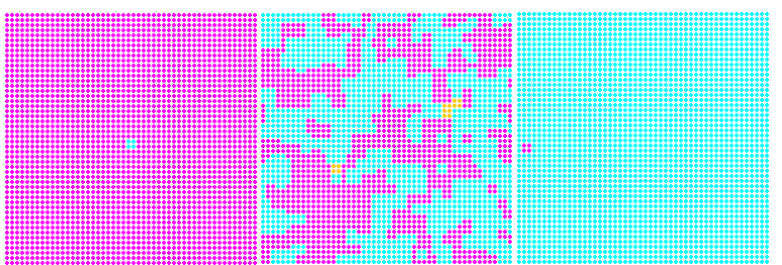
(a) $p(0)=0.125$
(b) $p(0)=0.5$
$p(0)=0.875$

: $\theta i=0.0$

: $0.0<\theta i<1.0$

: $\theta i=1.0$

(2) Preferences of agents (examples)

Fig. 3. Assignment of agents (case S-0.01) (a) $p(0)=0.125$

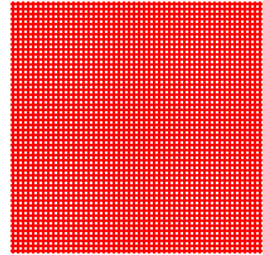

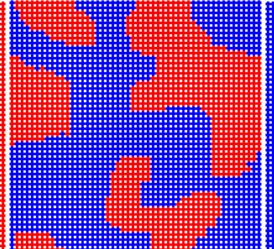

(b) $p(0)=0.5$

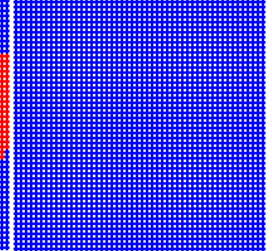

$p(0)=0.875$
:S1 : S2

(1) Behaviors of agents (examples)

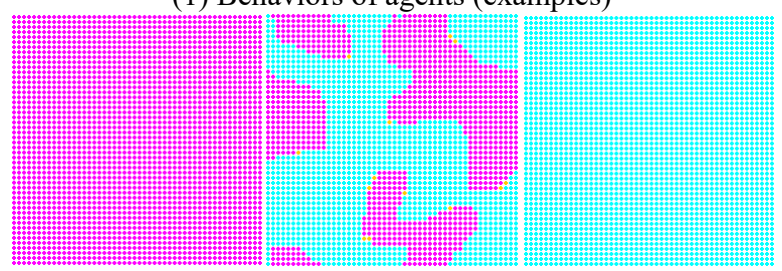
(a) $p(0)=0.125$
(b) $p(0)=0.5$
$p(0)=0.875$

: $\theta i=0.0 \bigcirc: 0.0<\theta i<1.0 \quad \bigcirc: \theta i=1.0$

(2) Preferences of agents (examples)

Fig. 4. Assignment of agents (case M-0.1)

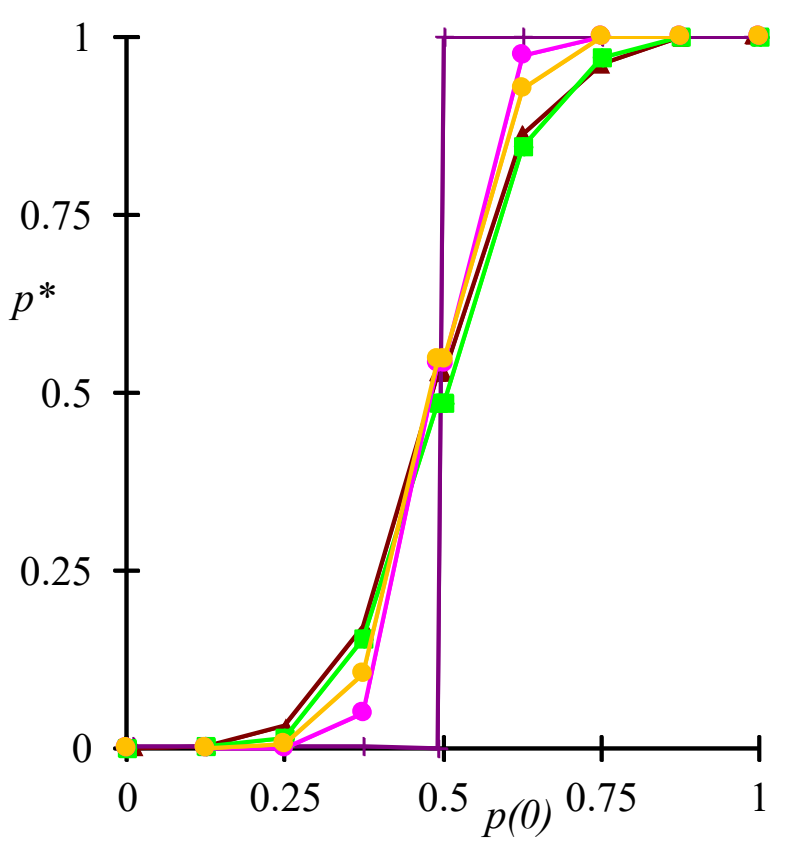

: M-0.01 : M-0.1 $\square:$ S-0.01 +: default M $\boldsymbol{\Delta}$ :default S

Fig. 5. Stability of collective behavior 


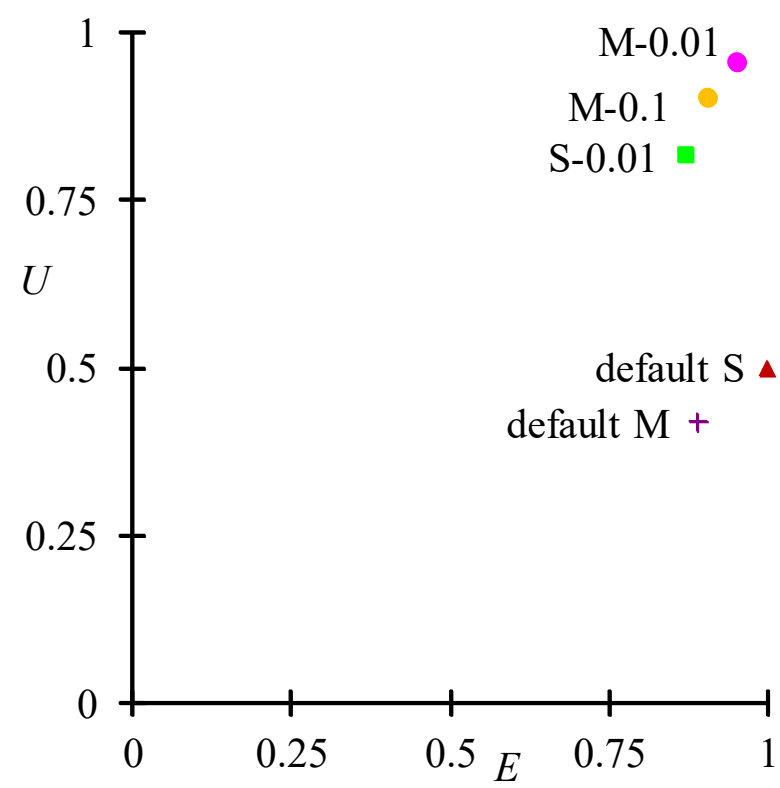

Fig. 6. Efficiency and equity $(p(0)=0.5)$

Efficiency and equity are shown in Fig. 6. They are lowest when the initial collective behavior $p(0)=0.5$ in all initial collective behaviors; thus, we show them as typical efficiency and equity in Fig. 6. We found that they increase in the order of S-0.01, M-0.1, and M-0.01. Efficiency and equity when the agent can change its partners are higher than when the agent cannot change partners. In addition, when delta $\Delta$ is 0.01 , efficiency and equity are higher than when delta $\Delta$ is 0.1 .

\section{Discussion}

Comparing M-0.01 and S-0.01, case S-0.01 is effective for the emergence of diversity. For case S-0.01, the agent cannot change partners and remains at the site. In other words, the interaction in population is isolated. However, the efficiency and equity of S- 0.01 are less than that of M-0.01. We found that there is a trade-off between the number of clustered interactions and utility in the population. M-0.01 corresponds to less clustered interaction because the agents can interact with distant agents. S-0.01 corresponds to isolated interaction because agents can only interact with neighbor agents. Our findings are consist with those of Axelrod et al. ${ }^{5}$, who showed that less clustered interaction make the world less diverse and suggested that an isolated area is required for the emergence of diversity. However, if population diversity emerges in less clustered interaction, the utility is greater than that of isolated interaction.
Comparing M-0.01 and M-0.1, the efficiency and equity of M-0.01 are greater than that of M-0.1. We found that it is effective to interact with partners with the same preferences for utility. In this study, each agent learns by two phases, i.e., best response and selfreinforcement. The learning speed when partners change is faster than that of self-reinforcement. Relative to self-reinforcement, M-0.01 demonstrate slow learning and M-0.1 demonstrate fast learning. Our results are consistent with those of Sandholm ${ }^{10}$ who shows the effectiveness of two different learning speed processes for the emergence of diversity. M-0.01 demonstrates different learning speed process than case M- 0.1 because the learning speeds of the two phases of $\mathrm{M}-0.01$ are very different than that of $\mathrm{M}-0.1$.

Thus, two-speed learning process and isolated interaction are required for emergence of diversity. However, the utility of less clustered interaction is greater than that of isolated interaction. Overall, isolated interaction is effective for the emergence of diversity. Tus, once sufficient diversity emerges, less clustered interaction is effective for the utility of each agent and the entire population.

\section{Conclusion}

The performance of the collective system strongly depends on the type of interaction and heterogeneity of preference. In this study, for the emergence of diversity, we found that two different learning speeds are required. In addition, isolated interaction is required for emergence of diversity. However, the utility of less clustered interaction is greater than that of isolated interaction. We found that isolated interaction is effective for the emergence of diversity. Then, once sufficient diversity emerges, less clustered interaction is effective for both the utility of each agent and the entire population.

\section{References}

1. C. Reynolds, Flocks, Herds, and Schools: A Distributed Behavioral Model, Computer Graphics (SIGGRAPH '87 Proceedings of the 14th annual conference on Computer graphics and interactive techniques) 21(4) (1987) 25-34.

2. K. Sims, Evolved Virtual Creatures, Computer Graphics (SIGGRAPH '94 Proceedings of the 21st annual conference on Computer graphics and interactive techniques) (1994) 15-22. 
3. D. Terzopoulos, X. Tu and R. Grzeszczuk, Artificial Fishes: Autonomous Locomotion, Perception, Behavior, and Learning in a Simulated Physical World, Artificial Life 1(4) (1994) 327-351.

4. M. Kubo and Y. Kakazu, Evaluation of Coordinated Motions of Multi - Agent Systems on Competition for Food between Ant Colonies, IPSJ Journal 35(8) (1994) 1555-1566.

5. R. Axelrod and M.D. Cohen, Harnessing Complexity (Basic Books, 2001).

6. M. Rubenstein, A. Cornejo and R. Nagpal, Programmable self-assembly in a thousand-robot swarm, Science 345(Issue 6198) (2041)795-799.

7. M. Sipper, Evolution of Parallel Cellular Machines: The Cellular Programming Approach (Springer-Verlag, Heidelberg, 1997).

8. F. Schweitzer (eds.), Interactive Structure Formation with Brownian Particles. Self-organization of complex structures: from individual to collective dynamics (Gordon and Breach Science Publishers, 1997) pp. 101118.

9. W.B. Arthur et al. (eds.), The economy as an interactive system. The Economy as a Complex Evolving System II (Addison Wesley, Reading, MA, 1997) pp. 491-533.

10. R. Axelrod, The Evolution of Cooperation (Basic Books, 1985).

11. W.H. Sandholm, Preference Evolution Two-Speed Dynamics and Rapid Social Change, Review of Economic Dynamics 4 (2001)637-679.
12. A. Rubinstein, Modeling Bounded Rationality (The MIT Press, 1998).

13. B. Huberman and N. Glance, Diversity and collective Action, Interdisciplinary Approaches to Nonlinear Complex Systems 62 of the series Springer Series in Synergetics (1993) 44-64.

14. T. Schelling, Micromotives and Macrobehavior (Norton, 1978).

15. W.B. Arthur, Inductive Reasoning and Bounded Rationality, American Economic Review 84(2) (1994) 406-411.

16. B. Fogel, K. Chellapia and P.J. Angeline, Inductive Reasoning and Bounded Rationality Reconsidered, IEEE Trans. or Evolutionary Computation 3 (2) (1999) 142146.

17. J. Hansarny and R. Selten, A Game Theory of Equilibrium Selection in Games (MIT Press, 1988).

18. D. Fudenberg and D. Levine D. The Theory of Learning in Games (The MIT Press, 1998).

19. M.A. Nowak, Evolutionary Dynamics: Exploring the Equations of Life (Belknap Press of Harvard University Press (2006).

20. S. Parsons et al. (eds.) Selecting Partners, Game Theory and Decision Theory in Agent-Based Systems, Multiagent systems, artificial societies, and simulated organizations: international book series, 5(Kluwer Academic, 2002) pp. $1-29$ 